\title{
Data-driven simulations of flank wear of coated cutting tools in hard turning
}

\author{
A. Cakan*, F. Evrendilek**, V. Ozkaner*** \\ *Mersin University, Dep. of Mechanical Engineering, Mersin, Turkey, E-mail: ahcakan@mersin.edu.tr \\ **Abant Izzet Baysal University, Dep. of Environmental Engineering, Bolu, Turkey, E-mail: fevrendilek@ibu.edu.tr \\ ***Mustafa Kemal University, Dep. of Electrical and Electronics Engineering, Antakya-Hatay, Turkey, \\ E-mail:vozkaner@mku.edu.tr
}

cross $^{\text {ref }}$ http://dx.doi.org/10.5755/j01.mech.21.6.12199

\section{Introduction}

Finish hard turning decreases machining time and complexity required to manufacture metal parts [1]. In finish hard turning, tool wear significantly affects surface quality and dimensional accuracy of machined steels which should be controlled within the desired limits for a given machining process as the most demanding customer requirements [2]. Development of real-time monitoring systems and continuous predictive models for temporal tool wear dynamics can improve cost effectiveness and productivity of finish hard turning for the manufacturing industry [3]. Numerous artificial neural networks (ANNs) and multiple non-linear regression (MNLR) models have been applied to predict flank wear dynamics. Sick [4], Ghosh et al. [5], Kamarthi et al. [6], Waibel et al. [7], Garg et al. [8], and Brezak et al. [9] found that multilayer perceptron (MLP) (52\%), recurrent neural network (RNN) (19\%), time-delay neural network (TDNN) (12\%), and radial basis function (RBF) $(7 \%)$ were the most frequently used supervised ANN topologies for a continuous estimation and real-time monitoring of wear and other tool conditions, with the remaining $10 \%$ such as support vector machine (SVM) belonging to the other supervised ANN topologies.

Predictive data-driven modeling includes multiple regressions, smoothing techniques, least squares, adaptive forecasting, stochastic time series and autoregressive moving average models, genetic algorithms, fuzzy logic, neural networks, and knowledge-based expert systems. So far, various ANNs have been applied successfully to real-time monitoring and predictions of tool conditions including wear under different machining operations and machine tools. The optimal ANN topology in the training process is generally determined by guesswork, some heuristics, and/or trial and error. Yang et al. [10] and Judd [11] established that configuration of ANNs in the training process is a nondeterministic polynomial time (NP)-complete problem. However, to our best knowledge, an extensive performance evaluation of different supervised ANN topologies has not been carried out for predicting flank wear.

The objective of this study was therefore to train, cross-validate, independently validate (test) and compare 28 ANNs and the best-fit MNLR model in predicting tool flank wear. In so doing, online real-time data of tool flank wear of the carbide inserts with the multilayer TiCN + $\mathrm{Al}_{2} \mathrm{O}_{3}+$ TiN coating were obtained from finish dry hard turning of AISI 52100 and AISI 4340 hardened steels in response to the three cutting speeds of 70, 98 and $142 \mathrm{~m} \mathrm{~min}^{-1}$ under the constant values of $0.027 \mathrm{~mm} \mathrm{~min}^{-1}$ for feed rate and $0.2 \mathrm{~mm}$ for cutting depth.

\section{Experimental design}

\subsection{Workpiece materials}

Workpieces in the form of round bars used in the finish dry hard turning experiment were $250 \mathrm{~mm}$ long with an external diameter of $60 \mathrm{~mm}$. The workpieces consisted of AISI 4340 medium-carbon, low-alloy and high-strength steel (HRC $53 \pm 1$ ) and AISI 52100 high-carbon, chromium alloy, high-strength, very high-hardness and abrasionresistance steel (HRC $56 \pm 1$ ). AISI 4340 hardened steel has a high strength martensitic steel with a wide range of applications to critical domains in aerospace engineering, and automotive transmissions. AISI 4340 hardened steel contains a typical chemical composition of $1.90 \% \mathrm{Ni}$, $0.92 \% \mathrm{Cr}, 0.71 \% \mathrm{Mn}, 0.43 \% \mathrm{C}, 0.38 \% \mathrm{Si}, 0.34 \% \mathrm{Mo}$, and balanced Fe. AISI 52100 steel typically consists of $1.5 \%$ $\mathrm{Cr}, 1.0 \% \mathrm{C}, 0.31 \% \mathrm{Mn}, 0.29 \% \mathrm{Si}, 0.03 \% \mathrm{Ni}, 0.003 \% \mathrm{P}$, $0.005 \% \mathrm{~S}$, and balanced $\mathrm{Fe}$. The experiments were conducted on a conventional lathe machine with a maximum spindle speed of $2000 \mathrm{r} / \mathrm{min}$ and a power of $5 \mathrm{~kW}$.

\subsection{Cutting inserts}

Carbide inserts of ISO designation CNMG 120404-WF with multilayer $\mathrm{TiCN}+\mathrm{Al}_{2} \mathrm{O}_{3}+\mathrm{TiN}$ coating through the medium temperature chemical vapor deposition (MTCVD) method were obtained from the manufacturer SandvikCoromant (Sweden). The tool holder used for the turning tests was DCLNR 2525M12 from SandvikCoromant (Sweden).

\subsection{Online real-time monitoring system}

The three cutting speeds (v) of 70, 98 and $142 \mathrm{~m} \mathrm{~min}^{-1}$ were selected to investigate their influence on flank wear in finish dry hard turning of AISI 4340 and AISI 52100 hardened steels under the constant values of $0.027 \mathrm{~mm} \mathrm{~min}^{-1}$ for feed rate and $0.2 \mathrm{~mm}$ for cutting depth. The turning conditions were selected according to the recommendations provided by the manufacturers of the cutting tools. The finish dry hard turning experiments were carried out for the fixed turning length of $250 \mathrm{~mm}$ in each run. During each run, the degree of flank wear of the coated carbide cutting tools was measured using an online realtime monitoring system by which the sensor voltage output was continuously recorded without the interruption of the turning operations based on a photo electronic sensor. 
The sensor consisted of a bifurcated optical fiber of $3 \mathrm{~mm}$ in diameter, a laser diode $(650 \mathrm{~nm} 20 \mathrm{~mW}$ visible red) as the light source, and a photodiode (IPL 10530 DAL) with amplification circuit as the detector. A schematic diagram of the experimental set-up is shown in Fig. 1, and this set-up ensures that any voltage change in the output signal is only due to change in intensity of the reflected light which can be correlated to flank wear. The cable carrying the output signal expressed in voltage (V) from the photodiode was connected to a data logger (HIOKI 8420-51 Memory Hilogger). The shortest sampling rate of 0.1 -second interval in the data logger was used in recording voltage data. The turning operation was stopped after the workpiece was turned with a fresh cutting tool for about $15 \mathrm{~mm}$ in length as an initial reference and was continued for the rest of the workpiece once the initial gap between the sensor and the workpiece was adjusted to $2.5 \mathrm{~mm}$. Change in the output voltage relative to the initial reference was measured during the turning operation.

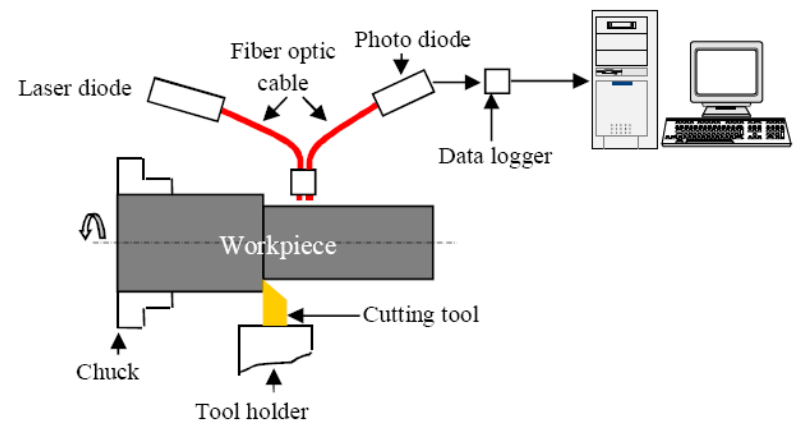

Fig. 1 A schematic overview of the experimental set-up including an automated real-time monitoring used in the present study

\subsection{Multiple non-linear regression models}

The Gaussian distribution (normally distributed errors) and heteroscedasticity (homogeneity of variance) assumptions were identified during the development of regression models based on Kolmogorov-Smirnov normality test, and a plot of standardized residuals versus the predicted values of $Y$, respectively. The normality assumption was not met at $P<0.01$. However, non-normality of the errors was considered to be addressed by the high sample size in the present study because the normality assumption is primarily of significance to robust inferences for small sample sizes [12]. No heteroscedasticity was encountered in the construction of regression models. Prior to regression and ANN modeling, the entire experimental dataset collected was randomly partitioned into training dataset $(n=11924)(60 \%)$, leave-one-out cross-validation dataset $(n=2981)(15 \%)$, and testing (independent validation) dataset $(n=4969)$ (25\%). Multiple non-linear regression models in which the estimation method is ordinary least squares were built in the following form:

$$
\begin{aligned}
F W_{T R}= & \beta_{o}+\beta_{1} t+\beta_{2} v+\beta_{3} W+\beta_{4} t^{2}+ \\
& +\beta_{5} v^{2}+\beta_{6} v W+\beta_{7} t v+\beta_{8} t W+\varepsilon,
\end{aligned}
$$

where $F W_{T R}$ is the measure of flank wear rate expressed in voltage $(\mathrm{V})$ for training dataset, $t$ is sampling time (s), $v$ is the cutting speed with three levels (70, 98 and $\left.142 \mathrm{~m} \mathrm{~min}^{-1}\right), W$ is the workpiece type with two categories $(1=$ AISI 52100 and $2=$ AISI 4340), and $\varepsilon$ is the residual error. The relationship between flank wear rate $(F W)$ in $\mathrm{mm}$ and $F W$ in $\mathrm{V}$ was found as follows:

$$
\begin{aligned}
& F W(\mathrm{~mm})=0.0061+0.0472 F W(\mathrm{~V}) \\
& \left(R^{2}=86.3 \% ; R M S E=0.003 ; n=9 ; P<0.001\right)
\end{aligned}
$$

The terms $\beta_{1}$ to $\beta_{8}$ refer to the coefficients of the explanatory variables, while $\beta_{0}$ is the intercept value. The interaction and quadratic terms were also used in the MNLR models. The best-fit MNLR model was chosen using the best subsets procedure in Minitab 16.1 software (Minitab Inc.). From all possible subsets of the explanatory variables, the best subsets procedure identifies the subset regression models and uses the highest values of coefficient of determination $\left(R^{2}\right)$ or adjusted coefficient of determination $\left(R_{a d j}^{2}\right)$, the lowest standard error $(S E)$ value, and a Mallows' $C_{p}$ value that is close to the number of explanatory variables plus the intercept as measures of model fit. Such a Mallows' $C_{p}$ value shows the model to be relatively precise and unbiased in estimating the true regression coefficients and predicting future responses [13].

\subsection{Supervised artificial neural network models}

The architecture of ANNs consists of input layer, output layer, hidden (intermediate) layer(s) with a number of interconnected processing elements (neurons), and the nature of the inter-neuron connections (learning algorithms). The strengths or weights of the inter-neuron connections in a given ANN are adjusted in training to achieve a desired behavior which depends on learning algorithm employed. ANNs can be structurally classified into feed forward and recurrent (feedback) networks and can be trained by supervised or unsupervised learning algorithms. Back propagation algorithm is an example of supervised learning algorithms.

The training dataset includes a subset of examples utilized only for learning. The cross-validation dataset refers to a subset of examples used to adjust the network parameters such as number of hidden layers and neurons, number of training cycles (epochs), and stopping criteria against the issue of over fitting in training. The testing (independent validation) dataset is a subset of examples not used in both training and cross-validation but in assessing the generalization performance of ANNs. In this study, the following 28 ANNs were built and compared using the NeuroSolution for Excel 6.3 software (Neuro Dimension Inc.): six MLP, three MLP with principal component analysis (MLPPCA), three generalized feedforward (GFF), three RBF, three TDNN, three time-lag recurrent network (TLRN), three RN, two linear regression (LR), one probabilistic neural network (PNN) and one SVM models (Table 1).

The number of hidden layers in the ANNs varied between zero and two which process and pass a weighted sum of the inputs through a non-linear function to the output layer. Individual weights were adapted progressively using the learning paradigm of either back propagation (BP) or back propagation through time (BPTT) algorithm in order to minimize the difference between calculated and expected outputs. Learning mode of the ANNs built in this study consisted of either online (stochastic) learning $(\mathrm{O})$ by 
which the weights were updated after every exemplar (exemplars/update $=1$ ) or batch (deterministic) learning (B) by which the weights are update after every epoch (exemplars/update $=$ exemplars/epoch). Gradient descent method employed as learning algorithm in the ANNs was either Momentum (M) or Levenberg-Marquardt (L). To update weights and bias, the $\mathrm{M}$ and $\mathrm{L}$ algorithms use the following equations, respectively [14]:

Table 1

Structural parameters used for the construction of 28 artificial neural networks (ANNs) in the present study

(Momentum rate set for all the ANNs in this study $=0.7$ )

\begin{tabular}{|c|c|c|c|c|c|c|}
\hline $\begin{array}{l}\text { ANN } \\
\text { topology }\end{array}$ & LP & $\begin{array}{l}\text { \# of } \\
\text { HL }\end{array}$ & LMA & TF in HL-OL & $\begin{array}{l}\# \text { of neu- } \\
\text { rons in } \\
\text { IL- HL- } \\
\text { OL }\end{array}$ & $\begin{array}{l}\text { LR in } \\
\text { IL-OL }\end{array}$ \\
\hline \multirow[t]{6}{*}{ MLP } & BP & 1 & O-M & TanhAxon & $16-10-26$ & $0.1-0.01$ \\
\hline & BP & 1 & B-L & TanhAxon & $9-5-1$ & 0.01 \\
\hline & BP & 1 & B-M & TanhAxon & $16-10-26$ & $1-0.1$ \\
\hline & BP & 2 & B-L & TanhAxon & $9-6-3-1$ & 0.01 \\
\hline & BP & 2 & O-M & TanhAxon & $9-12-6-1$ & $0.1-0.001$ \\
\hline & BP & 2 & B-M & TanhAxon & $9-12-6-1$ & $1-0.01$ \\
\hline \multicolumn{2}{|c|}{ MLPPCABP } & 1 & B-L & TanhAxon & $2-5-1$ & 0.01 \\
\hline & BP & 1 & O-M & TanhAxon & $2-10-1$ & $0.1-0.01$ \\
\hline & BP & 1 & B-M & TanhAxon & $2-10-1$ & $1-0.1$ \\
\hline \multirow[t]{2}{*}{ LR } & BP & 0 & B-M & BiasAxon & $16-26$ & 0.1 \\
\hline & BP & 0 & B-L & BiasAxon & $9-1$ & 0.01 \\
\hline \multirow[t]{3}{*}{$\overline{\mathrm{GFF}}$} & BP & 1 & B-L & TanhAxon & $9-10-1$ & 0.01 \\
\hline & BP & 1 & O-M & TanhAxon & $9-10-1$ & $0.1-0.01$ \\
\hline & $\mathrm{BP}$ & 1 & B-M & TanhAxon & $9-4-1$ & $1-0.1$ \\
\hline \multirow[t]{3}{*}{ RBF } & BP & 1 & B-L & $\begin{array}{l}\text { GaussianAxon- } \\
\text { TanhAxon }\end{array}$ & $-9-10-1$ & 0.01 \\
\hline & BP & 1 & O-M & $\begin{array}{l}\text { GaussianAxon- } \\
\text { TanhAxon }\end{array}$ & $-9-20-1$ & 0.01 \\
\hline & $\mathrm{BP}$ & 1 & B-M & $\begin{array}{l}\text { GaussianAxon- } \\
\text { TanhAxon }\end{array}$ & $-9-20-1$ & 0.1 \\
\hline PNN & BP & 0 & $\mathrm{~N}-\mathrm{N}$ & GaussianAxon & 9-235-1 & 0.01 \\
\hline SVM & BP & 0 & $\mathrm{~N}-\mathrm{N}$ & GaussianAxon & $2-4-1$ & 0.01 \\
\hline \multirow[t]{3}{*}{ TDNN } & BPTT & 1 & B-L & TanhAxon & $9-4-1$ & 0.01 \\
\hline & BPTT & 1 & O-M & TanhAxon & $9-10-1$ & $0.1-0.01$ \\
\hline & BPTT & 1 & B-M & TanhAxon & 9-10-1 & $1-0.1$ \\
\hline \multirow[t]{3}{*}{ TLRN } & BPTT & 1 & B-L & TanhAxon & $2-4-1$ & 0.01 \\
\hline & BPTT & 1 & O-M & TanhAxon & 2-10-1 & $0.1-0.01$ \\
\hline & BPTT & 1 & B-M & TanhAxon & 2-10-1 & $1-0.1$ \\
\hline \multirow[t]{3}{*}{$\mathrm{RN}$} & BPTT & 1 & B-L & TanhAxon & $9-4-1$ & 0.01 \\
\hline & BPTT & 1 & O-M & TanhAxon & $9-10-1$ & $0.1-0.01$ \\
\hline & BPTT & 1 & B-M & TanhAxon & 9-10-1 & $1-0.1$ \\
\hline
\end{tabular}

MLP: Multilayer Perceptron; MLPPCA: MLP with Principal Component Analysis; LR: Linear Regression; GFF: Generalized Feedforward; PNN: Probabilistic Neural Network; SVM: Support Vector Machine; TDNN: Time-Delay Network; TLRN: TimeLag Recurrent Network; RN: Recurrent Network; LP: learning paradigm; LMA: learning mode-algorithm; TF: transfer function; LR: learning rate; BP: backpropagation; BPTT: backpropagation through time; O: online; B: batch; M: momentum; L: LevenbergMarquardt; IL: input layer; HL: hidden layer; OL: output layer; and $\mathrm{N}$ : none.

$$
\left.\begin{array}{c}
\Delta w_{\mathrm{ji}}(n)=\alpha \Delta w_{\mathrm{ji}}(n-1)+n \frac{\partial E}{\partial w_{j i}} ; \\
w_{k+1}=w_{k}-\left[J^{T} J+\lambda I\right]^{-1} J^{T} e,
\end{array}\right\}
$$

where $\Delta w_{\mathrm{ji}}(n)$ is the correction applied to the synaptic weight connecting neuron $i$ to neuron $j ; \alpha$ is momentum parameter; $\eta$ is learning rate; $E$ is the error function; $w$ is weight; $J$ is Jacobian matrix containing first derivatives of the network errors with respect to the weights and biases, $e$ is a vector of network errors; $I$ is identity matrix; $\lambda$ is learning parameter; $J^{T}$ is transpose matrix of $J$; and $J^{T} J$ is Hessian matrix.

The three types of transfer functions (Axons) used in the hidden and output layers of the ANNs were TanhAxon, BiasAxon, and GaussianAxon through which the input data are scaled and shifted to fit the range of the Axon and then the output data are computed with the current weights. The TanhAxon applies a bias and non-linear tanhfunction (hyperbolic tangent) to each neuron in the layer. BiasAxon is a linear axon with adjustable slope and adaptable bias. The GaussianAxon applies a Gaussian function in the range of 0 to 1 to each neuron in the layer and is considered to be a local function approximator since it only responds significantly to the peak of the Gaussian of the input space. The BiasAxon component as a linear component adds a bias term in the range of -1 to 1 to each neuron. The TanhAxon applies a scaled and biased hyperbolic tangent function in the range of -1 to 1 to each neuron in the layer.

Performance statistics used for the processes of training, cross-validation and testing of ANNs were mean absolute error (MAE), and $R^{2}(\%)$ as follows:

$$
\begin{aligned}
& M A E=\frac{1}{n} \sum_{i=1}^{n}\left|\left(x_{p i}-x_{d i}\right)\right| ; \\
& R^{2}=1-\frac{\sum_{i=1}^{n}\left(x_{p i}-x_{d i}\right)^{2}}{\sum_{i=1}^{n}\left(x_{p i}-\bar{x}\right)^{2}},
\end{aligned}
$$

where $x_{p i}$ and $x_{d i}$ are the predicted and measured outputs from observation $i$, respectively. $\bar{x}$ is the average value of the measured output, and $n$ is the number of data.

Mean absolute error is a measure of how close predictions are to the experimental measurements and is more robust to outliers than is mean squared error. The default number of iterations for training was set at 1000 . Momentum rate was set at 0.7 in all the ANNs, while learning rate ranged from 1 to 0.001 depending on the input or output layer of a given ANN. The training process was performed for 100 epochs without improvement in cross-validation MAE for all the ANNs except for LR-0-B$\mathrm{L}$ (for five epochs) and PNN-0-N-N (for three epochs). Fisher's Least Significant Difference (LSD) test following one-way analysis of variance (ANOVA) was used to detect significant differences among the mean performance metrics of the 28 ANNs in terms of the learning paradigms, number of hidden layers, the learning modes, the learning algorithms, and the ANN topologies, based on the training, cross-validation and testing at the significance level $(P<0.05)$.

\section{Results and discussion}

3.1. Data-driven modeling of temporal dynamics of flank wear

The direction, rate and amount of temporal trends 
of flank wear were depicted in response to turning time for each of the workpiece types (4340 versus 52100) and the cutting speeds in Fig. 2. Regardless of the workpiece types and the cutting speeds applied, there was an increasing trend in flank wear with the increasing turning time. The regression lines indicate that the rate of increase in flank wear was highest at $98 \mathrm{~m} \mathrm{~min}^{-1}$ regardless of the workpiece types applied. For workpiece 52100, flank wear did not clearly increase as a function of the cutting speed and was highest at $98 \mathrm{~m} \mathrm{~min}^{-1}$ relative to the other lowest or highest cutting speeds. For workpiece 4340, the highest cutting speed $\left(142 \mathrm{~m} \mathrm{~min}^{-1}\right)$ resulted in the highest flank wear (Fig. 2). The best-fit MNLR model based on the training dataset elucidated $58.2 \%$ of variation in flank wear as a function of time, the three cutting speeds, the two workpieces, the quadratic terms of time and the cutting speeds, and the interaction terms of the cutting speeds, the workpieces, and time as follows:

$$
\left.\begin{array}{c}
F W_{T R}(\mathrm{~V})=4.068-0.0002 t(\mathrm{~s})-0.02 v\left(\mathrm{~m} \mathrm{~min}^{-1}\right)+ \\
+0.012 W-0.000001 t^{2}+0.00009 v^{2}+ \\
+0.001 v W+0.000005 t v+0.0008 t W \\
\left(S E=0.191 ; R_{\text {adj }}^{2}=58.2 \% ; n=11924 ; P<0.001\right)
\end{array}\right\}
$$

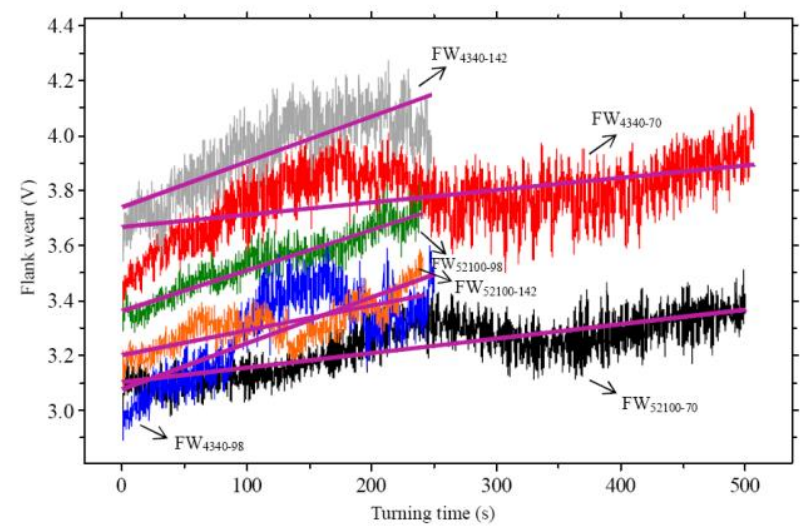

Fig. 2 Rate of change in flank wear $(F W)$ measured in voltage $(\mathrm{V})$ via a real-time monitoring system in response to turning time $(t)$, the three cutting speeds (70, 98 and $142 \mathrm{~m} \mathrm{~min}^{-1}$ ), and the two workpiece types (4340 versus 52100). The best-fit temporal linear regression lines found are as follows: $F W_{4340-142}=3.741+0.00163 t \quad\left(R^{2}=65.8 \%\right) ;$ $F W_{4340-70}=3.669+0.000445 t \quad\left(R^{2}=28.8 \%\right)$; $F W_{52100-98}=3.362+0.00147 t \quad\left(R^{2}=86.8 \%\right)$; $F W_{52100-142}=3.201+0.000909 t \quad\left(R^{2}=57.6 \%\right)$; $F W_{4340-98}=3.075+0.00168 t \quad\left(R^{2}=59.1 \%\right) ; \quad$ and $F W_{52100-70}=3.102+0.000529 t\left(R^{2}=58.6 \%\right)$

The sign and magnitude of the coefficients of the predictors indicate direction and rate of change in flank wear, respectively, in response to a one-unit increase in a given predictor with the other predictors being held constant. Based on the cross-validation and testing of the bestfit MNLR model, the comparison of measured versus predicted values resulted in $R^{2}$ of $58.5 \% \quad(S E=0.189$; $n=2981 ; \quad P<0.001)$ and $R^{2}$ of $59.5 \% \quad(S E=0.143$; $n=4969 ; \quad P<0.001)$, respectively. Testing results for measured versus predicted values are given in Fig. 3. Indi- vidual $S E, T$ and $P$ statistics of the predictors of the best-fit MNLR model are presented for the training, crossvalidation and testing datasets in Table 2. Except for the predictors of time and workpiece type, the inclusion of all the individual predictors in the best-fit MNLR model was significant $(P=0.001)$ (Table 2$)$.

The performance metrics of MAE and $R^{2}$ based on the training, cross-validation and testing datasets pointed to PNN-0-N-N, MLP-2-O-M, and MLP-2-B-L as the best three models and to RN-1-B-L, MLPCA-1-B-M, and TLRN-1-B-L as the worst three models out of the 28 ANNs, respectively (Table 3 ). The best ANNs significantly outperformed the best MNLR model in terms of all the performance metrics used. Based on each of the three datasets, Fisher's LSD test was used to compare the mean performance metrics of the 28 ANNs in terms of the following five factors: (1) BP versus BPTT learning paradigm, (2) number of hidden layers, (3) B versus O learning mode, (4) M versus L learning algorithm, and (5) ANN topologies with different number of hidden layers.

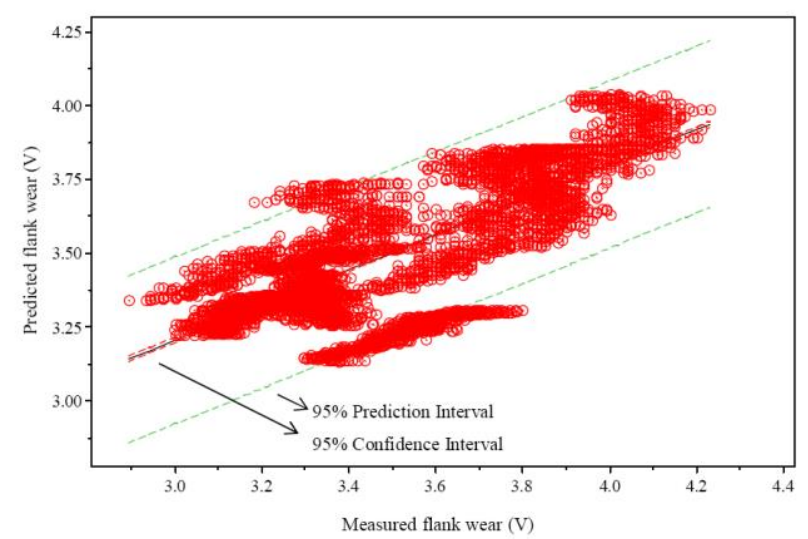

Fig. 3 A comparison of measured versus predicted flank wear expressed in voltage (V) based on testing dataset $\left(R^{2}=59.5 \% ; S E=0.143 ; n=4969 ; P<0.001\right)$

Table 2

Individual results of the best-fit multiple non-linear regression model based on training, cross-validation and testing datasets

\begin{tabular}{|l|r|r|r|r|r|r|}
\hline \multirow{3}{*}{$\begin{array}{l}\text { Predic- } \\
\text { tor }\end{array}$} & \multicolumn{2}{|c|}{$\begin{array}{c}\text { Training } \\
(n=11924)\end{array}$} & \multicolumn{2}{c|}{$\begin{array}{c}\text { Cross-validation } \\
(n=2981)\end{array}$} & \multicolumn{2}{c|}{$\begin{array}{c}\text { Testing } \\
(n=4969)\end{array}$} \\
\cline { 2 - 7 } & \multicolumn{1}{|c|}{$S E$} & \multicolumn{1}{c|}{$P$} & \multicolumn{1}{c|}{$S E$} & $P$ & \multicolumn{1}{c|}{$S E$} & $P$ \\
\hline Intercept & 0.047 & 0.001 & 0.094 & 0.001 & 0.071 & 0.001 \\
\hline$t$ & 0.0001 & 0.017 & 0.0002 & 0.438 & 0.0002 & 0.161 \\
\hline$v$ & 0.001 & 0.001 & 0.001 & 0.001 & 0.001 & 0.001 \\
\hline $\mathrm{W}$ & 0.016 & 0.419 & 0.032 & 0.266 & 0.024 & 0.829 \\
\hline$t^{2}$ & $1.2^{*} 10^{-7}$ & 0.001 & $2.4^{*} 10^{-7}$ & 0.001 & $1.8 * 10^{-7}$ & 0.001 \\
\hline$v^{2}$ & $3.4^{*} 10^{-6}$ & 0.001 & $6.9 * 10^{-6}$ & 0.001 & $5.2 * 10^{-6}$ & 0.001 \\
\hline$v^{*} \mathrm{~W}$ & 0.0001 & 0.001 & 0.0002 & 0.001 & 0.0002 & 0.001 \\
\hline$t^{*} v$ & $8.6^{*} 10^{-7}$ & 0.001 & $1.7 * 10^{-6}$ & 0.002 & $1.3 * 10^{-6}$ & 0.001 \\
\hline$t^{*} \mathrm{~W}$ & $2.9^{*} 10^{-5}$ & 0.001 & $5.8^{*} 10^{-5}$ & 0.001 & $4.4^{*} 10^{-5}$ & 0.001 \\
\hline
\end{tabular}

$t$ : time (s); $v$ : cutting speed $\left(\mathrm{m} \mathrm{min}^{-1}\right) ; W$ : workpiece type

The mean performance metrics significantly differed only in terms of ANN topologies with different number of hidden layers. For example, a multiple comparison of the mean performance metrics of the testing dataset was presented in terms of 11 ANN topologies with different number of hidden layers in Table 4 . The $R^{2}$ values of RN1 , and MLPPCA-1 were significantly less than those of 
PNN-0, MLP-1, MLP-2, GFF-1, RBF-1, and TDNN-1 ( $P$ $=0.004)$. The MAE values of MLPPCA-1, RN-1, and SVM-0 were significantly greater than those of PNN-0, MLP-1, MLP-2, GFF-1, RBF-1, and TDNN-1 $(P=0.005)$.

Table 3

Performance statistics of 28 artificial neural networks (ANNs) used in the present study

\begin{tabular}{|l|c|c|c|c|c|c|}
\hline \multirow{2}{*}{ ANN model } & \multicolumn{2}{|c|}{$\begin{array}{c}\text { Training } \\
(n=11924)\end{array}$} & \multicolumn{2}{c|}{$\begin{array}{c}\text { Cross- } \\
\text { validation } \\
(n=2981)\end{array}$} & $\begin{array}{c}\text { Testing } \\
(n=4969)\end{array}$ \\
\cline { 2 - 8 } & \multicolumn{7}{|c|}{ MAE } & \multicolumn{1}{|c|}{$R^{2}$} & \multicolumn{1}{|c|}{ MAE } & $R^{2}$ & MAE & $R^{2}$ \\
\hline MLP-1-O-M & 0.047 & 96.08 & 0.046 & 96.12 & 0.046 & 96.12 \\
\hline MLP-1-B-L & 0.048 & 95.74 & 0.048 & 95.72 & 0.048 & 95.73 \\
\hline MLP-1-B-M & 0.057 & 94.01 & 0.057 & 93.80 & 0.056 & 93.99 \\
\hline MLP-2-B-L & $\mathbf{0 . 0 4 5}$ & $\mathbf{9 6 . 2 6}$ & $\mathbf{0 . 0 4 4}$ & $\mathbf{9 6 . 2 7}$ & $\mathbf{0 . 0 4 4}$ & $\mathbf{9 6 . 3 1}$ \\
\hline MLP-2-O-M & $\mathbf{0 . 0 4 3}$ & $\mathbf{9 6 . 5 0}$ & $\mathbf{0 . 0 4 4}$ & $\mathbf{9 6 . 4 2}$ & $\mathbf{0 . 0 4 3}$ & $\mathbf{9 6 . 5 3}$ \\
\hline MLP-2-B-M & 0.128 & 73.01 & 0.126 & 72.40 & 0.129 & 71.44 \\
\hline MLPPCA-1-B-L & 0.161 & 52.43 & 0.159 & 52.02 & 0.163 & 49.76 \\
\hline MLPPCA-1-O-M & 0.185 & 40.51 & 0.180 & 41.32 & 0.184 & 37.96 \\
\hline MLPPCA-1-B-M & 0.253 & 5.36 & 0.249 & 4.73 & 0.249 & 5.36 \\
\hline LR-0-B-M & 0.163 & 51.88 & 0.160 & 51.71 & 0.164 & 49.41 \\
\hline LR-0-B-L & 0.163 & 51.91 & 0.161 & 51.68 & 0.165 & 49.47 \\
\hline GFF-1-B-L & 0.045 & 96.16 & 0.045 & 96.15 & 0.045 & 96.17 \\
\hline GFF-1-O-M & 0.046 & 96.25 & 0.045 & 96.29 & 0.045 & 96.30 \\
\hline GFF-1-B-M & 0.119 & 75.03 & 0.118 & 74.47 & 0.120 & 73.72 \\
\hline RBF-1-B-L & 0.081 & 88.13 & 0.081 & 87.74 & 0.079 & 88.15 \\
\hline RBF-1-O-M & 0.053 & 94.73 & 0.054 & 94.48 & 0.053 & 94.67 \\
\hline RBF-1-B-M & 0.110 & 81.87 & 0.109 & 81.36 & 0.109 & 81.08 \\
\hline PNN-0-N-N & $\mathbf{0 . 0 4 2}$ & $\mathbf{9 6 . 6 3}$ & $\mathbf{0 . 0 4 2}$ & $\mathbf{9 6 . 5 9}$ & $\mathbf{0 . 0 4 2}$ & $\mathbf{9 6 . 6 4}$ \\
\hline SVM-0-N-N & 0.293 & 89.72 & 0.237 & 79.79 & 0.243 & 78.07 \\
\hline TDNN-1-B-L & 0.051 & 95.33 & 0.051 & 95.28 & 0.050 & 95.36 \\
\hline TDNN-1-O-M & 0.046 & 96.17 & 0.046 & 96.16 & 0.046 & 96.14 \\
\hline TDNN-1-B-M & 0.115 & 76.52 & 0.115 & 75.70 & 0.115 & 75.42 \\
\hline TLRN-1-B-L & 0.220 & 21.59 & 0.216 & 22.75 & 0.219 & 20.83 \\
\hline TLRN-1-O-M & 0.045 & 96.28 & 0.045 & 96.25 & 0.045 & 96.27 \\
\hline TLRN-1-B-M & 0.161 & 53.70 & 0.160 & 52.76 & 0.164 & 50.96 \\
\hline RN-1-B-L & 0.308 & 1.26 & 0.304 & 1.15 & 0.308 & 1.47 \\
\hline RN-1-O-M & 0.166 & 51.86 & 0.163 & 51.55 & 0.167 & 49.27 \\
\hline RN-1-B-M & 0.165 & 50.77 & 0.162 & 50.69 & 0.166 & 48.49 \\
\hline & & & & & & \\
\hline
\end{tabular}

Table 4

A multiple comparison of mean performance metrics of testing dataset in terms of 11 ANN topologies with different number of hidden layers based on Fisher's Least Significant Difference (LSD) test following one-way analysis of variance (ANOVA)

\begin{tabular}{|l|l|l|l|l|}
\hline \multicolumn{1}{|c|}{ Model-HL } & $n$ & \multicolumn{1}{c|}{$R^{2}$} & \multicolumn{1}{c|}{ Model-HL } & \multicolumn{1}{c|}{ MAE } \\
\hline PNN-0 & 1 & $96.64^{\mathrm{a}}$ & PNN-0 & $0.042^{\mathrm{bc}}$ \\
\hline MLP-1 & 3 & $95.28^{\mathrm{a}}$ & MLP-1 & $0.050^{\mathrm{c}}$ \\
\hline TDNN-1 & 3 & $88.97^{\mathrm{a}}$ & GFF-1 & $0.070^{\mathrm{bc}}$ \\
\hline GFF-1 & 3 & $88.73^{\mathrm{a}}$ & TDNN-1 & $0.070^{\mathrm{bc}}$ \\
\hline MLP-2 & 3 & $88.10^{\mathrm{a}}$ & MLP-2 & $0.072^{\mathrm{bc}}$ \\
\hline RBF-1 & 3 & $87.97^{\mathrm{a}}$ & RBF-1 & $0.080^{\mathrm{bc}}$ \\
\hline SVM-0 & 1 & $78.07^{\mathrm{ab}}$ & TLRN-1 & $0.142^{\mathrm{ab}}$ \\
\hline TLRN-1 & 3 & $56.02^{\mathrm{ab}}$ & LR-0 & $0.164^{\mathrm{ab}}$ \\
\hline LR-0 & 2 & $49.44^{\mathrm{ab}}$ & MLPPCA-1 & $0.198^{\mathrm{a}}$ \\
\hline RN-1 & 3 & $33.08^{\mathrm{b}}$ & RN-1 & $0.213^{\mathrm{a}}$ \\
\hline MLPPCA-1 & 3 & $31.03^{\mathrm{b}}$ & SVM-0 & $0.243^{\mathrm{a}}$ \\
\hline
\end{tabular}

HL: number of hidden layers. $R^{2}$ and MAE values that do not share the same letter are significantly different at $P$ values of 0.004 and 0.005 , respectively.

\subsection{Evaluation of data-driven simulations of flank wear}

Palanisamy et al. [15] predicted tool wear of a carbide cutter using a universal milling machine on AISI 1020 steel with training $R^{2}$ values of $99.5 \%$ for MLP-3 and 98.6\% for the best-fit MNLR model based on cutting speed, feed rate, depth of cut, their quadratic terms, and their interaction terms. Onwubolu et al. [16] reported that enhanced-group method of data handling approach (eGMDH) with three hidden layers outperformed a polynomial neural network to predict wear on the end mill cutter in dry machining mild steel blocks as a result of cutting speed, feed rate, and depth of cut, based on a validationbased $R^{2}$ value of $80.5 \%$. Al Hassani [17] used MLP-1 and regression model to quantify tool wear of cemented carbide inserts in turning titanium alloy (Ti-6Al-4V) based on cutting speed, feed rate, depth of cut, coolant, cutting forces, and vibration and found validation-based $R^{2}$ values of $78.9 \%$ and $72.5 \%$, respectively.

Ghani et al. [18] modeled flank tool wear of $\mathrm{Cu}$ bic Boron Nitride (CBN) insert in dry turning titanium alloy (Ti-6Al-4V) using a MNLR model based on cutting speed, depth of cut, and feed rate with training-based $R^{2}$ value of $82 \%$. Ali and Dhar [19] modeled wear of uncoated carbide insert in turning medium-carbon steel under minimum quantity lubrication using an ANN with BP paradigm and L learning algorithm as a function of cutting speed, feed rate, depth of cut, and marching time which yielded a validation-based $R^{2}$ value of $96.4 \%$. MNLRbased modeling of wear of PVD TiN/TiCN/TiN-coated carbide insert in the machining of a nimonic $\mathrm{C}-263$ nickelbase alloy resulted in validation-based $R^{2}$ values of $69.5 \%$ for an exponential model and $99.9 \%$ for a polynomial model as a function of cutting speed, coolant concentration, depth of cut, and feed rate [20]. Ranganathan and Senthilvelan [21] developed MNLR models of wear on the rake face of tungsten carbide inserts due to hot turning of AISI 316 stainless steel as a function of cutting speed, feed rate, depth of cut, and their interaction terms under three temperatures whose validation-based $R^{2}$ values were $88.8 \%$ for $200^{\circ} \mathrm{C}, 83.7 \%$ for $400^{\circ} \mathrm{C}$, and $44.3 \%$ for $600^{\circ} \mathrm{C}$. As Siddhpura and Paurobally [22] stated, the development of a universal approach with the integration of processbased and data-driven models and monitoring systems can overcome the limitations imposed by neural networks techniques, and application-specific nature of most tool condition monitoring systems.

Wang et al. [23] found that Gaussian mixture regression model with an $R^{2}$ of $95.2 \%$ outperformed back propagation neural network, RBF network, and multiple linear regression (MLR) in the prediction of the tool wear for a titanium alloy Ti-6Al-4V workpiece. Slamani et al. [24] developed tool wear and cutting force prediction models in the trimming of carbon fibre reinforced polymers, with the exponential model having the highest $R^{2}$ of 93\%. Das et al. [25] predicted surface roughness with an $R^{2}{ }_{a d j}$ of $97.5 \%$ in turning of hardened AISI 4140 steel with PVD-TiN coated mixed $\mathrm{Al}_{2} \mathrm{O}_{3}+\mathrm{TiCN}$ ceramic inserts under dry environment based on a response surface method. Palanikumar and Davim [26], and Seeman et al. [27] quantified tool wear on the machining of glass fibre reinforced polymer composites, and metal matrix composites with $R^{2}$ adj values of $95.7 \%$, and $98.2 \%$, respectively. Bhattacharyya et al. [28] introduced such signal processing techniques as discrete wavelet transform, time-domain averaging, isotonic regression, and exponential smoothing to estimate tool wear in face milling operation from the 
acquired force signals and found that it is possible to achieve wear estimation within industrially acceptable limits for a complex and intermittent milling operation with a higher accuracy by an appropriate signal processing technique than the earlier methods using complex signal processing and ANNs.

\section{Conclusions}

An online real-time monitoring system was used for the acquisition of experimental data of flank wear on the multilayer-coated carbide inserts due to finish dry hard turning of the two workpieces under the three cutting speeds. The collected data were randomly partitioned in order to train, cross-validate and test the 28 ANNs as a function of time, cutting speed, and type of workpiece materials and the best-fit MNLR model as a function of time, cutting speed, type of workpiece materials, their quadratic terms, and their interaction terms. A multiple comparison of the mean performance metrics of the ANNs based on LSD test was carried out using the three datasets in terms of BP versus BPTT learning paradigm, number of hidden layers, $\mathrm{B}$ versus $\mathrm{O}$ learning mode, $\mathrm{M}$ versus $\mathrm{L}$ learning algorithm, and topologies with different number of hidden layers.

Out of the 28 supervised ANNs, the ten ANNs (three RN, three MLPPCA, two LR and two TLRN models) fell behind the best-fit MNLR regression in terms of the predictive power based on the three datasets for better predictions for tool flank wear. PNN, MLP-2-O-M and MLP-2-B-L were the best three models, respectively, thus outperforming the rest of the 28 ANNs in terms of all the performance metrics explored. A multiple comparison of the ANNs based on LSD test revealed that the mean performance metrics used differed significantly only in 11 topologies. Given all the performance metrics based on each of the training, cross-validation and testing datasets, PNN appeared to be the best ANN model. The MLP-1 topology appeared to stand out in the ANNs with the highest $R^{2}$ and the lowest MAE as particularly different from the RN-1 and MLPPCA-1 topologies with the lowest $R^{2}$ and the highest MAE across all the datasets. A performance evaluation of predictive data-driven modeling using online real-time monitoring signals, and signal processing techniques can be also extended to the other types of machining operations such as drilling and milling and of machining variables such as surface roughness and workpiece dimension, and the selection of the optimal cutting conditions such as feed rate and depth of cut in the future studies.

\section{Acknowledgement}

This work was funded by the Scientific Research Projects Unit (BAP 809.03.279) of Abant Izzet Baysal University.

\section{References}

1. Kumar, C.H.R.V.; Ramamoorthy, B. 2007. Performance of coated tools during hard turning under minimum fluid application, Journal of Materials Processing Technology 185: 210-216.

http://dx.doi.org/10.1016/j.jmatprotec.2006.03.148.
2. Huang, Y.; Chou, Y.K.; Liang, S.Y. 2007. CBN tool wear in hard turning: a survey on research progresses, International Journal of Advanced Manufacturing Technology 35: 443-453. http://dx.doi.org/10.1007/s00170-006-0737-6.

3. Choudry, S.K.; Bartarya, G. 2003. Role of temperature and surface finish in predicting tool wear using neural network and design of experiments, International Journal of Machine Tools and Manufacture 43: 747-753. http://dx.doi.org/10.1016/S0890-6955(02)00166-9.

4. Sick, B. 2002. On-line and indirect tool wear monitoring in turning with artificial neural networks: a review of more than a decade of research, Mechanical Systems and Signal Processing 16: 487-546.

http://dx.doi.org/10.1006/mssp.2001.1460.

5. Ghosh, N.; Ravi, Y.B.; Patra, A.; Mukhopadhyay, S.; Paul, S.; Mohanty, A.R.; Chattopadhyay, A.B. 2007. Estimation of tool wear during CNC milling using neural network-based sensor fusion, Mechanical Systems and Signal Processing 21: 466-479. http://dx.doi.org/10.1016/j.ymssp.2005.10.010.

6. Kamarthi, S.V.; Kumara, S.R.T.; Cohen, P.H. 2000. Flank wear estimation in turning through wavelet representation of acoustic emission signals, Journal of Manufacturing Science and Engineering, Transactions of the ASME 122: 12-19.

http://dx.doi.org/10.1115/1.538886.

7. Waibel, A.; Hanazawa, L.; Hinton, G.; Shikano, K.; Lang, K.J. 1989. Phoneme recognition using timedelay neural networks, IEEE Transactions on Acoustics, Speech, and Signal Processing 37: 328-339. http://dx.doi.org/10.1109/29.21701.

8. Garg, S.; Patra, K.; Khetrapal, V.; Pal, S.K.; Chakraborty, D. 2010. Genetically evolved radial basis function network based prediction of drill flank wear, Engineering Applications of Artificial Intelligence 23: 1112-1120. http://dx.doi.org/10.1016/j.engappai.2010.02.012.

9. Brezak, D.; Majetic, D.; Udiljak, T.; Kasac, J. 2012. Tool wear estimation using an analytic fuzzy classifier and support vector machines, Journal of Intelligent Manufacturing 23: 797-809.

http://dx.doi.org/10.1007/s10845-010-0436-x.

10. Yang, W.A.; Zhou, W.; Liao, W.; Guo, Y. 2014. Prediction of drill flank wear using ensemble of coevolutionary particle swarm optimization basedselective neural network ensembles, Journal of Intelligent Manufacturing. http://dx.doi.org/10.1007/s10845-013-0867-2

(in press).

11. Judd, J.S. 1987. Learning in networks is hard, In: IEEE Proc. the First International Conference on Neural Networks (San Diego), IEEE, New York, 685-692.

12. Williams, M.N.; Grajales, C.A.G.; Kurkiewicz, D. 2013. Assumptions of multiple regression: correcting two misconceptions, Practical Assessment, Research \& Evaluation 18: 1-14.

13. Mallows, C.L. 1973. Some comments on Cp, Technometrics 15: 661-675.

14. Bishop, C.M. 1995. Neural Networks for Pattern Recognition, Clarendon Press, Oxford, 482 p.

15. Palanisamy, P.; Rajendran, I.; Shanmugasundaram, S. 2008. Prediction of tool wear using regression and 
ANN models in end-milling operation, International Journal of Advanced Manufacturing Technology 37: 29-41.

http://dx.doi.org/10.1007/s00170-007-0948-5.

16. Onwubolu, G.C.; Buryan, P.; Lemke, F. 2008. Modeling tool wear in end-milling using enhanced GMDH learning networks, International Journal of Advanced Manufacturing Technology 39: 1080-1092. http://dx.doi.org/10.1007/s00170-007-1296-1.

17. AlHassani, A.R. 2013. Modeling of tool wear when turning of Ti-6Al-4V titanium alloy, M.Sc. Thesis UAE: the American University of Sharjah.

18. Ghani, J.A.; Rizal, M.; Sayuti, A.; Nuawi, M.Z.; Rahman, M.N.Ab.; Haron, C.H.C. 2009. New regression model and I-Kaz method for online cutting tool wear monitoring, World Academy of Science, Engineering and Technology 36: 420-425.

19. Ali, S.M.; Dhar, N.R. 2010. Modeling of tool wear and surface roughness under MQL condition-a neural approach, Canadian Journal on Artificial Intelligence, Machine Learning \& Pattern Recognition 1: 7-25.

20. Ezugwu, E.O.; Olajire, K.A.; Bonney, J. 2001. Modelling of tool wear based on component forces, Tribology Letters 11: 55-60. http://dx.doi.org/10.1023/A:1016651430877.

21. Ranganathan, S.; Senthilvelan, T. 2010. Optimizing the process parameters on tool wear of WC insert when hot turning of AISI 316 stainless steel, ARPN Journal of Engineering and Applied Sciences 5: 24-35.

22.Siddhpura, A.; Paurobally, R. 2013. A review of flank wear prediction methods for tool condition monitoring in a turning process, International Journal of Advanced Manufacturing Technology 65: 371-393. http://dx.doi.org/10.1007/s00170-012-4177-1.

23. Wang, G.; Qian, L.; Guo, Z. 2013. Continuous tool wear prediction based on Gaussian mixture regression model, International Journal of Advanced Manufacturing Technology 66: 1921-1929. http://dx.doi.org/10.1007/s00170-012-4470-z.

24. Slamani, M.: Chatelain, J-F.; Hamedanianpour, $\mathbf{H}$ 2015. Comparison of two models for predicting tool wear and cutting force components during high speed trimming of CFRP, International Journal of Material Forming 8: 305-316. http://dx.doi.org/10.1007/s12289-014-1170-2.

25. Das, S.R.; Kumar, A.; Dhupal, D. 2015. Surface roughness analysis of hardened steel using TiN coated ceramic inserts, International Journal of Machining and Machinability of Materials 17: 22-38. http://dx.doi.org/10.1504/IJMMM.2015.069217.
26. Palanikumar, K.; Davim, J.P. 2007. Mathematical model to predict tool wear on the machining of glass fibre reinforced plastic composites, Materials \& Design 28: 2008-2014. http://dx.doi.org/10.1016/j.matdes.2006.06.018.

27. Seeman, M.; Ganesan G.; Karthikeyan R.; Velayudham, A. 2010. Study on tool wear and surface roughness in machining of particulate aluminum metal matrix composite-response surface methodology approach, International Journal of Advanced Manufacturing Technology 48: 613-624.

http://dx.doi.org/10.1007/s00170-009-2297-z.

28. Bhattacharyya, P.; Sengupta, D.; Mukhopadhyay, S. 2007. Cutting force-based real-time estimation of tool wear in face milling using a combination of signal processing techniques, Mechanical Systems and Signal Processing 21: 2665-2683. http://dx.doi.org/10.1016/j.ymssp.2007.01.004.

\section{A. Cakan, F. Evrendilek, V. Ozkaner}

\section{DATA-DRIVEN SIMULATIONS OF FLANK WEAR OF} COATED CUTTING TOOLS IN HARD TURNING

S u m m a r y

Insurance of surface quality and dimensional tolerances in finish hard turning necessitates the development of accurate predictive models. This study aimed at modeling flank wear of multilayer-coated carbide inserts in finish dry hard turning of AISI 4340 and AISI 52100 hardened steels based on 28 artificial neural networks (ANNs) and the best-fit multiple non-linear regression (MNLR) model. Online-monitored flank wear of multilayer-coated carbide inserts was modeled as a function of the three cutting speeds of 70, 98 and $142 \mathrm{~m} \mathrm{~min}^{-1}$, and the two workpieces under the constant feed rate and cutting depth of $0.027 \mathrm{~mm}$ $\mathrm{min}^{-1}$ and $0.2 \mathrm{~mm}$, respectively. Out of the 28 ANNs, 18 ANNs appeared to be capable of better predictions for tool flank wear than the best-fit MNLR model. Probabilistic neural network (PNN) outperformed all the remaining models based on all the training, cross-validation and testing dataset-related performance metrics.

Keywords: carbide tools, online monitoring, data-driven modeling, finish turning.

Received May 05, 2015

Accepted September 17, 2015 